\title{
LETTER \\ Efficient Motion Vector Re-Estimation Based on a Novel Cost Model for a H.264/AVC Transcoder
}

\author{
Soongi HONG ${ }^{\dagger \mathrm{a})}$, Nonmember, Yoonsik $\mathrm{CHOE}^{\dagger \mathrm{b})}$, Member, and Yong-Goo $\mathrm{KIM}^{\dagger \dagger \mathrm{c})}$, Nonmember
}

\begin{abstract}
SUMMARY In transcoding, it is well known that refinement of the motion vectors is critical to enhance the quality of transcoded video while significantly reducing transcoding complexity. This paper proposes a novel cost model to estimate the rate-distortion cost of motion vector composition in order to develop a reliable motion vector re-estimation method that has reasonable computation cost. Based on a statistical analysis of motion compensated prediction errors, we design a basic form of the proposed cost model as a function of distance from the optimal motion vector. Simulations with a transcoder employing the proposed cost model demonstrate a significant quality gain over representative video transcoding schemes with no complexity increase.

key words: H.264/AVC, transcoding, motion vector re-estimation, motion vector cost estimator
\end{abstract}

\section{Introduction}

The recent explosive deployment of smart devices poses a challenge for creating a seamless multimedia service across all such terminals, which have different computation and display capabilities. To meet the demands for high quality of experience (QoE) over such varying devices, dynamic adaptive streaming via HTTP has been standardized by 3GPP [1] and MPEG [2], [3], etc. These standards basically provide mechanisms to change service quality according to network conditions, and large scale service provisioning can be easily realized because they are based on the standard HTTP protocol. However, changes in service quality require a number of predefined media presentations (MPs); it is difficult for this number to be large because this MP corresponds to a copy of an encoded media stream at a certain quality level. Therefore, speedy and reliable transcoding has regained a lot of attention in order to support a higher quality of seamless multimedia service. In this paper we focus on H.264/AVC video transcoding which involves resolution changes for accommodating the variety of terminal displays.

Such transcoding involves resolution changes [4], [5], and it is well known that the composition and refinement

Manuscript received August 13, 2015.

Manuscript revised October 18, 2015.

Manuscript publicized December 4, 2015.

${ }^{\dagger}$ The authors are with Department of Electrical \& Electronics Engineering, Yonsei University, Shinchon-dong, Seoul 120-749, South Korea.

${ }^{\dagger \dagger}$ The author is with Department of Media Engineering, Korean German Institute of Technology, Sangam-dong, Seoul 121-270, South Korea.

a)E-mail: s82.hong@samsung.com

b) E-mail: yschoe@yonsei.ac.kr

c) E-mail: ygkim@ @git.ac.kr

DOI: $10.1587 /$ transinf.2015EDL8181 of motion vectors (MVs) are very important because they directly affect the quality of transcoded video and can also significantly reduce the complexity of transcoding. In order to quickly find an appropriate MV for each prediction unit of a reduced resolution video, there have been various MV re-estimation (MVRE) approaches that combine the corresponding MVs of higher resolution.

In order to develop a reliable MVRE method without an excessive increase in computations, this paper proposes a novel cost model to estimate the rate-distortion (RD) cost of MV composition and employs H.264/AVC transcoder with it.

The remainder of this paper is organized as follows. Section 2 explains the proposed cost model for the MVRE of the H.264/AVC transcoder. Section 3 presents an MVRE method based on the proposed cost model. Section 4 provides the simulation results. Finally, Sect. 5 concludes this paper.

\section{The Proposed Rate-Distortion Cost Model for Mo- tion Vector Composition}

To obtain a relationship between the optimal $\mathrm{MV} m v_{i}$ and the motion compensated prediction (MCP) error, $D_{i}, D_{i}$ is plotted against distance $l, l=\pi(d m v)=\pi\left(m v-m v_{i}\right)$, where $\pi$ is distance measure and $d m v$ is the motion vector difference (MVD) between any MV, $m v$, and the optimal MV, $m v_{i}$. For confirmation of these relationships, we calculate the differential distortion $\Delta D_{i} / \Delta l$ using a JM encoder [6] with a fixed QP. Based on these experimental results, we assume that the differential distortion can be denoted by

$$
\frac{d D_{i}}{d l} \simeq \frac{\Delta D_{i}}{\Delta l}=a \frac{c_{i}}{l}
$$

which depicts the proportional relation between the $\Delta D_{i} / \Delta l$ and $c_{i}$ and the inverse-proportional relation between the $\Delta D_{i} / \Delta l$ and $l$.

Using (1) and assuming $D_{i}(l)$ to be integrable $l>0$,

$$
D_{i}(l)=a c_{i} \int \frac{1}{l} d l= \begin{cases}a c_{i} \ln (l)+b & \text { if } l>0, \\ c_{i} & \text { if } l=0\end{cases}
$$

with $a$ and $b$ parameterizing the functional relationship between distance, $l$, and distortion, $D_{i}(l)$. These parameters come from simulations using a variety of test sequences and described in Table 1.

For bits spent on the MVs, $R$ can be written as 
Table 1 Model parameters of the proposed rate-distortion cost estimator for motion vector re-estimation

\begin{tabular}{ccrrrr}
\hline \hline $\begin{array}{c}\text { Model } \\
\text { Param. }\end{array}$ & $\begin{array}{c}\text { MCP } \\
\text { Mode }\end{array}$ & QP22 & QP27 & QP32 & QP27 \\
\hline \multirow{4}{*}{ a } & P16x16 & 0.270 & 0.176 & 0.110 & 0.068 \\
& P16x8 & 0.222 & 0.175 & 0.122 & 0.077 \\
& SMB8x8 & 0.290 & 0.220 & 0.152 & 0.099 \\
& SMB8x4 & 0.319 & 0.261 & 0.188 & 0.131 \\
& SMB4x4 & 0.386 & 0.321 & 0.239 & 0.170 \\
\hline \multirow{6}{*}{ b } & P16x16 & 47970.726 & 54673.469 & 70479.474 & 94136.002 \\
& P16x8 & 21883.706 & 27772.913 & 37053.554 & 48359.039 \\
& SMB8x8 & 13441.495 & 15997.214 & 20363.250 & 27043.093 \\
& SMB8x4 & 7723.545 & 9752.059 & 12649.936 & 16757.108 \\
& SMB4x4 & 4638.107 & 5744.370 & 7352.676 & 9550.192 \\
\hline \hline
\end{tabular}

$$
R(m v)=\operatorname{mvd} 2 \operatorname{bits} \operatorname{Tb}\left[m v-p m v_{p}\right],
$$

where $m v$ is the desired MV of current blocks, $p m v_{p}$ is the predicted motion vector (PMV) extracted from neighborhood MBs of pre-coded video, and "mvd2bitsTb" is the conversion table for the motion vector difference (MVD), $m v-p m v_{p}$, to the bits which are a result of entropy coding.

Using (2) and (3), our proposed MV cost estimator is defined as

$$
f_{i}(m v)=D_{i}\left(\pi\left(m v-m v_{i}\right)\right)+\lambda_{\text {motion }} R(m v),
$$

where $m v$ is the desired MV of current blocks, $m v_{i}$ is the the optimal MV of the current block, and $\pi$ is the distance measure. To use the MV cost estimator in (4), the distance measure $\pi$ has to be defined properly. In this paper, $\pi$ is mathematically induced from simple assumptions. In the H.264/AVC, an image is segmented and encoded into blocks of variable block-sizes from $4 \times 4$ to $16 \times 16$. Being small enough to compare the size of an image and properly estimated by MCP, we assume that these segmented image blocks have the monotonous MCP error. Since the MCP error increases with concentric circles in the this case, the distance measure has the equivalent and independent weights of horizontal and vertical direction. Thus, the distance measure can be defined as

$$
l=\pi\left(m v-m v_{i}\right)=\pi\left(d m v_{i}\right)=\left|d m v_{x}\right|+\left|d m v_{y}\right|,
$$

where $d m v_{x}$ and $d m v_{y}$ are the horizontal component and vertical component of the MVD $d m v_{i}$, respectively.

We evaluate the correctness of the proposed cost estimation model and the model parameters. First of all, we divide the motion vector domain centered around $m v_{i}$ into four sub-region to examine the regional impacts. And then, we calculate the mean absolute percentage error (MAPE) between the estimated rate-distortion costs and the conventional H.264/AVC rate-distortion costs. The MAPE in each sub-region is calculated as

$$
E_{R_{d}}=\frac{1}{N_{R_{d}}} \sum_{m v \in R_{d}}\left|\frac{f_{i}(m v)-j(m v)}{j(m v)}\right|,
$$

where $N_{R_{d}}$ is the cardinality of the sub-region set $R_{d}=$ $\{m v: 4(d-1) \leq l<4 d, d \in\{1,2,3,4\}\}, f_{i}(m v)$ is the estimated rate-distortion cost at $m v$, and $j(m v)$ is the calculating values from the H.264/AVC rate-distortion equation at $m v$.
Table 2 The mean absolute percent error of the proposed cost estimation model comparing with H.264/AVC at each sub-region and four QPs.

\begin{tabular}{ccrrr|r}
\hline \hline QP & $R_{1}$ & $R_{2}$ & $R_{3}$ & $R_{4}$ & Avg. \\
\hline QP22 & 0.225 & 0.163 & 0.215 & 0.259 & 0.216 \\
QP27 & 0.189 & 0.153 & 0.209 & 0.257 & 0.202 \\
QP32 & 0.142 & 0.134 & 0.195 & 0.251 & 0.181 \\
QP37 & 0.096 & 0.111 & 0.174 & 0.239 & 0.155 \\
\hline Avg. & 0.163 & 0.140 & 0.198 & 0.252 & 0.188 \\
\hline \hline
\end{tabular}

Table 2 depicts the MAPE of the proposed cost estimator at each sub-region and four QPs. Generally speaking, the rate-distortion functions of high $\mathrm{QP}$ values tend to become simpler than that of low QP values. Thus, the MAPE is decreased with larger QP value. In aspect of regional impacts, the MAPEs of $R_{1}$ and $R_{2}$ are significantly less than that of other regions. Our proposed cost estimation model can work as a good guide for the motion vector re-estimation because the the average MAPE of all of sub-regions and QPs is only 0.188 .

\section{Motion Vector Re-Estimation Based on the Pro- posed Cost Model}

Let $m v_{i}$ be the MV, $m d_{i}$ be the MCP mode, and $c_{i}$ be the MCP error from the $i$ th corresponding MB partition. Thus, the context sets extracted from pre-coded bitstream are a set of $K$ corresponding MVs $V=\left\{m v_{1}, m v_{2}, \cdots, m v_{K}\right\}$, a set of K corresponding MCP errors $D=\left\{c_{1}, c_{2}, \cdots, c_{K}\right\}$, and a set of K corresponding MCP modes $M=\left\{m d_{1}, m d_{2}, \cdots, m d_{K}\right\}$. For the proposed cost estimator, we have to define the corresponding region which is spatially related to the current $\mathrm{MB}$ of low resolution domain (transcoded bitstream) in high resolution domain (pre-coded bitstream) and the downsizing factor as described in Fig. 1. As there are at most $K \mathrm{MVs}$, the estimated MV cost $J$ can be obtained as

$$
\begin{aligned}
J(m v)= & \frac{1}{S} \sum_{i=1}^{K} \frac{s_{i}^{I}}{s_{i}^{I}+s_{i}^{O}} f_{i}(m v) \\
= & \frac{1}{S} \sum_{i=1}^{K} \eta_{i} D_{i}\left(\pi\left(m v-m v_{i}\right)\right)+\lambda_{\text {motion }} K R(m v) \\
& \eta_{i}=\frac{s_{i}^{I}}{s_{i}^{I}+s_{i}^{O}}, \quad S=\sum_{i=1}^{K} \eta_{i}
\end{aligned}
$$

where $m v_{i} \in V$ is a MV from the $i$ th corresponding MB partition of the pre-coded H.264/AVC video, $c_{i} \in D$ is a distortion of a corresponding MV $m v_{i}, s_{i}^{I}$ is the size of MCP mode in the corresponding region, $s_{i}^{O}$ is the size of MCP mode out of corresponding region, $\eta_{i}$ is an area-weighted strength of $i$ th corresponding MB mode, and $f_{i}(m v)$ is designated in (4). In the case of downsizing by an integer factor, $\eta_{i}$ is always one because $s_{i}^{O}$ is always zero which means that a whole area of MCP mode is included in a corresponding region. Note that if a corresponding partition is coded with skip mode in a precoded H.264/AVC video, then its MV is set to the one predicted from the MVs of previously coded partitions. Also, if a corresponding partition is intra-coded, it 
will not be involved in estimating the new MV. For this reason, if all the corresponding partitions are intra-coded, the new MB partition will also be transcoded with intra mode.

As described in Fig. 1, the proposed algorithm can estimate the MV cost of current P16x16 MB and SMB modes if the corresponding regions are defined in accordance with various downsizing factors and inter modes. Thus, our proposed algorithm can support all of inter modes without a SKIP mode, which means that P16x16, P16x8, P8x16, P8x8, SMB8x8, SMB8x4, SMB4x8, and SMB4x4 are supported by our proposed algorithm.

Using (7), we can estimate the cost of any given MV $m v$. The search pattern, the set $S$ of the MVs, needs to be defined for serving MVs to the proposed MV cost estimator. If the set $S$ whose cardinality $L$ is defined as $S=\left\{m v_{s}\right.$ : $s=1, \cdots, L\}$, the estimated cost set $E$ is represented as $E=\left\{J\left(m v_{s}\right): s=1, \cdots, L\right\}$ where $J(\cdot)$ is the proposed cost estimator in (7). Then, let $E^{\mathrm{TO}}$ be a totally ordered set of $E$, which is denoted by

$$
E^{\mathrm{TO}}=\left\{J\left(m v_{k}\right): J\left(m v_{k}\right) \leq J\left(m v_{k+1}\right), m v_{k} \in S\right\},
$$

where $k=1, \cdots, L$. Because the elements of $E^{\mathrm{TO}}$ are estimated costs of given MVs and totally ordered, we can establish a set $C$ of the re-estimated MV candidates by getting the MVs associated with the lower index elements of the set $E^{\mathrm{TO}}$. Therefore, a set $C$ is defined as

$$
C=\left\{m v_{k}: J\left(m v_{k}\right) \in E^{\mathrm{TO}}, k=1, \cdots, N\right\},
$$

where $N \leq L$. Consequently, a set $C$ is a subdivision of $S$ into non-overlapping, nonempty subsets and has MVs associated with the $N$ th minimum estimated costs of $E^{\mathrm{TO}}$. It is known that the search pattern has important influence on speed and distortion performance in block motion estimation. Generally speaking, the large cardinality of set $S$

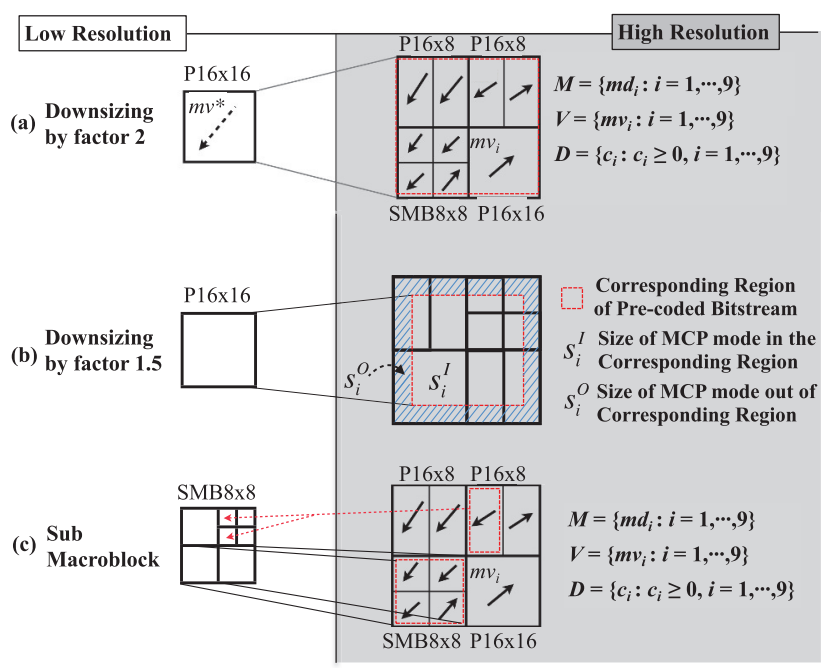

Fig. 1 Mapping schemes of corresponding region for a current macroblock depending on the downsizing factors and inter modes of (a) P16x16 with downsizing factor 2, (b) P16x16 with downsizing factor 1.5, and (c) SMB with downsizing factor 2 . can provide good coding efficiency, but they spend a lot of time testing the search points in the set $S$. So, to balance speed and distortion, we propose the corresponding MV centered local search strategy. For example, if the current MB has the three corresponding MVs $m v_{i} \in V$ in the precoded H.264/AVC video and the local search points $m v_{r} \in P$ with 1 integer-pixel resolution, the total number of search points $(L)$ is 27 which is equal to the size of the product set $S=V \times P$. Then, if the four MVs are used for candidates of a re-estimated MV $(N=4)$, the MVs with the estimated cost $J\left(m v_{k}\right) \in E^{\mathrm{TO}}$ from first to fourth are picked as the candidates $m v_{k} \in C$ from the MVs of $S$. Finally, we select a re-estimated MV from the MV candidates and their \pm 1 quarter-pixel neighborhoods by the Lagrangian cost function of H.264/AVC in the fractional MV refinement process. Because a target application of this letter is the motion vector re-estimation scheme, the inter mode selection method is same as that of the H.264/AVC JM reference software. The only difference between these two methods is that the MV estimation of H.264/AVC is replaced by the proposed cost estimation based motion vector re-estimation (CEMVR).

\section{Simulation Results}

We conducted a sets of experiments to evaluate and compare the performance of the proposed CEMVR method against other existing methods for transcoding pre-coded H.264/AVC videos to downsized H.264/AVC videos. The detailed information about the test sequences and encoding conditions are listed in Table 3. The sequences were encoded in the H.264/AVC format with IPPP GOP structure, and then transcoded to new sequences at a 4-to-1 reduced frame size using the cascaded H.264/AVC Full-Decoding and Full-Encoding (FDFE) scheme, the existing H.264/AVC transcoding schemes (AWMVM [4] and KMCMV [5]), and the proposed H.264/AVC transcoding scheme (CEMVR). Full-search fractional (half- and quarter-pixel accuracy) ME with \pm 32 as the search range and the infinite GOP size are used in the proposed, KMCMV, AWMVM and FDFE techniques. For objective comparison, the BD-PSNR and BD-RATE [7] are computed with respect to the sequentially processed video by decoding of pre-encoded bitstream and applying a downsampling filter to it, where the BD-PSNR and BD-RATE are the percentage of increasing PSNR at

\begin{tabular}{|c|c|c|c|c|}
\hline Sequences & Resolution & Motion Type & FPS & No. Frames \\
\hline SpeedBag & \multirow{8}{*}{$\begin{array}{c}\text { FHD } \\
(1920 \times 1080)\end{array}$} & & 30 & 570 \\
\hline ElephantsDream & & Regular & 24 & 1569 \\
\hline BigBuckBunny & & Motion & 24 & 1431 \\
\hline BluSky & & & 25 & 217 \\
\hline Factory & & & 30 & 1339 \\
\hline PedestrianArea & & Irregular & 25 & 375 \\
\hline SintelTrailer & & Motion & 24 & 1253 \\
\hline Tractor & & & 25 & 690 \\
\hline \multicolumn{5}{|c|}{ H.264/AVC Encoding Conditions } \\
\hline Main Profile & 1 Ref. Frame & $\begin{array}{l}\text { Full Search } \\
\text { Mode }\end{array}$ & $\begin{array}{l}32 \text { MV Search } \\
\text { Range }\end{array}$ & RDO On \\
\hline
\end{tabular}

Table 3 Test sequences and simulation conditions 
Table 4 BD-Bitrates obtained by transcoding H.264/AVC precoded sequences

\begin{tabular}{clccc}
\hline \hline Pre & & \multicolumn{3}{c}{ BD-Bitrate (\%) } \\
\cline { 3 - 5 }$\downarrow$ & Sequence & & & Proposed \\
Trans & & AWMVM & KMCMV & CEMVR \\
\hline & SpeedBag & 13.50 & 13.21 & 3.94 \\
& ElephantsDream & 10.99 & 10.54 & 3.98 \\
$1920 \times 1080$ & BigBuckBunny & 7.40 & 7.17 & 0.98 \\
$\downarrow$ & BluSky & 7.34 & 6.76 & 1.38 \\
\cline { 2 - 5 } $960 x 540$ & Factory & 50.17 & 48.41 & 23.41 \\
& PedestrianArea & 27.26 & 26.02 & 7.89 \\
& SintelTrailer & 23.65 & 23.61 & 5.38 \\
& Tractor & 31.30 & 29.65 & 10.46 \\
\hline 1080p Regular Motion & 9.81 & 9.42 & 2.57 \\
1080p Irregular Motion & 33.10 & 31.92 & 11.79 \\
\hline \multicolumn{2}{r}{ Overall Average } & 21.45 & 20.67 & 7.18 \\
\hline \hline
\end{tabular}

the same bitrate and increasing bitrate at the same PSNR, respectively. The simulation was conducted on a PC with an Intel Core 2 Quad Q6600 2.4GHz, and 12GB of DDR3 RAM. Note that all the techniques are implemented based upon the H.264/AVC recommendations and JM17.2 reference software.

Table 4 shows BD-Bitrates. A positive value in the table indicates an increase in bit-rates for the BD-Bitrates compared to the FDFE method. Obviously the BD-Bitrate values in the table are almost positive because the FDFE method has the most superior performance compared to other methods in terms of compression efficiency. The performance of the proposed CEMVR (i.e., with four candidates and 1 quarter-pixel refinement) technique in terms of BD-Bitrate (see Table 4) outperforms the AWMVM (with 1 quarter-pixel refinement) and KMCMV (with four candidates and 1 quarter-pixel refinement) techniques due to the accuracy of the cost estimation model for MV selection (see Sect. 2) and MVRE procedure based on multiple candidate MVs (see Sect. 3). We recommend the proposed CEMVR technique for speeding up the entire encoding time with enhanced rate-distortion performance compared to other conventional techniques. The KMCMV, which has the multiple candidate MVs, demonstrated a meaningful gain $(1.18 \%$ BD-Bitrate reduction) over the AWMVM for 1080p irregular motion test sequences, but the overall gain $(0.78 \%$ BDBitrate reduction) is marginal since it cannot fully use the existing information of a pre-encoded bitstream to find candidate MVs, as discussed in Sect. 2. In contrast with the KMCMV, the proposed CEMVR achieves a significant gain over the AWMVM not only for 1080p irregular motion test sequences but also for overall test sequences. The gains of BD-Bitrate reduction are $21.31 \%$ and $14.27 \%$, respectively. Moreover, compared with KMCMV, the proposed CEMVR still captures a lot of BD-Bitrate reduction which is $13.49 \%$ on the average of all test sequences. Specifically, a BDBitrate reduction of the proposed CEMVR about the factory sequence is far superior to that of the KMCMV, which is up to $25 \%$. This is the experimental evidence of getting significantly better RD performance in test sequences with irregular motion as discussed in Sect. 2 because a factory sequence has many objects that move in random or complex ways.

From the literature, we have found that the AWMVM proposed by Tan et al. [4] is the best one considered both accuracy and complexity. The AWMVM reduces on average $77 \%$ of the computational time but suffers RD performance degradation for irregular motion video sequences and fast regular motion video sequences. We have compared the computational complexity of our algorithm with this well-known MVRE algorithm. We have also compared our result with the recent algorithm (KMCMV).The proposed CEMVR technique reduces $71 \% \sim 88 \%$ of the computational complexity on average compared to the FDFE, whereas the AWMVM and KMCMV techniques reduce $71 \% \sim 88 \%$ and $64 \% \sim 85 \%$ compared to the FDFE, respectively. The significant gain of BD-rates is from the efforts of the proposed cost model because coding gain is preserved in any combinations of the local search range and multiple candidates compared with two existing schemes. The complexity reduction is depend on the effort of the local search range. The number of multiple candidates is the fine tuning factor for a balance of coding gain and complexity reduction. We decided that the local search range and the number of multiple candidates are set at 1 and 4 .

\section{Conclusions}

In this paper, we proposed a novel MV cost estimator for reestimating the MVs in a H.264/AVC transcoder. Since our proposed cost estimator can fully utilize the existing information of a pre-coded bitstream, we can estimate the cost of any given MV and select reliable MVs as candidate MVs by comparing their estimated cost in the MVRE process. As a result, compared to representative known schemes, the transcoder employing the MVRE algorithm by the proposed cost estimator leads to a significant quality gain without a critical complexity increment, especially for complex motion videos. Furthermore, our proposed cost estimator can easily be applied to any type of MVRE scheme if the bitstream is pre-coded.

\section{References}

[1] "3GPP; Technical Specification Group Services and System Aspects; Transparent end-to-end Packet-switched Streaming Service (PSS); Progressive Download and Dynamic Adaptive Streaming over HTTP (3GP-DASH)," no.26.247, 2010.

[2] "Dynamic adaptive streaming over http (dash)," FCD 23001-6, ISO/IEC JTC 1/SC 29/WG 11 (MPEG), Daegu, Jan. 2011.

[3] I. Sodagar, "The mpeg-dash standard for multimedia streaming over the internet," MultiMedia, IEEE, vol.18, no.4, pp.62-67, April 2011.

[4] Y.-P. Tan and H. Sun, "Fast motion re-estimation for arbitrary downsizing video transcoding using h.264/avc standard," IEEE Transactions on Consumer Electronics, vol.50, no.3, pp.887-894, 2004.

[5] K. Kim, S. Hong, and Y. Choe, "Efficient motion re-estimation method based on k-means clustering for spatial resolution reduction transcoding," Picture Coding Symposium (PCS), 2012, pp.221-224, May 2012.

[6] K. Suehring, "H.264/avc reference software."

[7] G. Bjøntegaard, "Calculation of average PSNR differences between RD-curves,” ITU-T SG16/Q.6 VCEG Doc., VCEG-M33, April 2001. 